\title{
Juego ampliado, juego restringido Producciones simbólicas tempranas en contextos de desigualdad*
}

\author{
Play Elaborated, Play Restricted \\ Early Symbolic Productions that Reproduce Inequality
}

Recibido: octubre 24 de 2013 | Revisado: febrero 22 de 2014 | Aceptado: abril 10 de 2014

Patricia Eliana Castillo Gallardo **

Universidad Diego Portales, Santiago, Chile

doi.org/10.11144/Javeriana.UPSY13-4.jajr

Para citar este artículo: Castillo, P. E. (2014). Juego ampliado, juego restringido. Producciones simbólicas tempranas en contextos de desigualdad. Universitas Psychologica, 13(4), PAG-PAG. http:// dx.doi.org/10.11144/Javeriana.UPSY13-4.jajr

\footnotetext{
* Agradecimientos:

** Facultad de Psicología. Correo electrónico: patricia. castillo@udp.cl
}

\begin{abstract}
RES U MEN
La indagación de las consecuencias de la desigualdad ha sido un importante tema de investigación desde hace varios años. Sin embargo, pocos estudios han abordado este tema desde la perspectiva de comprender cuáles de las consecuencias encontradas inciden directamente en la reproducción y perpetuación de una determinada estratificación social. Esta investigación se desarrolló en Santiago de Chile y se propuso indagar en la infancia temprana las características del juego simbólico, con la finalidad de acceder a las formas tempranas de subjetivación y comprender desde ahí las diferencias en la identificación y en la transmision transgeneracional de contenidos que explicarían la reproducción de la desigualdad. Utilizando la metodología de observación clínica del juego infantil, se observaron niños de 3 a 5 años en el espacio educacional y familiar. Se identificaron siete dimensiones de diferenciación, con las cuales se describen las caracteristicas de dos modalidades de juego: ampliado y restringido.

Palabras clave

juego infantil; desigualdad; observación clínica; infancia; fenómenos sociales
\end{abstract}

\section{A B S T R A C T}

The inquiry into consequences of inequality has been an important research topic for several years to date. However, few studies have addressed this issue from the perspective of understanding which consequences found affect directly the reproduction and maintenance of a certain social stratification. This research aimed to investigate the characteristics of symbolic play in early childhood (3-5 years) in Santiago de Chile, order to access the earliest forms of subjectivity and understand from their differences in identification and transmission transgenerational content which explain the reproduction of inequality. Using the methodology of clinical observation of children's play, children 3 to 5 years were observed in the educational and family space. Seven dimensions of differentiation with which the characteristics of two modes of play were identified.

Keywords:

children's game; inequality; clinical observation; children; social phenomena 


\section{Introducción}

La desigualdad y sus efectos sobre la infancia ha sido abordada en numerosos estudios (Özkan, 2010; Yoshikawa, Aber, \& Beardslee, 2012); algunos de ellos han llegado a afirmar desastrosas consecuencias cognitivas, a causa de los factores estresantes asociados a la pobreza (Today, 2009). Felizmente, no pocos investigadores en el mundo han salido al paso de esta última afirmación, comprobando las serias dificultades que dichos estudios tienen tanto en la definicion de la población que se estudia como en la forma de aislar los factores con los que fundamentan sobredeterminación del aspecto socioeconómico en el curso del desarrollo cognitivo de los niños (McLeod, Nonnemaker, \& Thiede Call, 2004; Niles \& Peck, 2008; Ready, 2010).

Así mismo, varios autores han demostrado el papel fundamental de la educación en lo que se refiere al desarrollo de competencias especificamente cognitivas y otras no cognitivas que resultan ser inclusive más relevantes a la hora de predecir el estatus social futuro (Janet, 2011; Lee, 2009; Magnuson, Meyers, Ruhm, \& Waldfogel, 2004; Mier \& Rabell, 2002).

Pareciera ser que en aquello que explica la reproducción social tienen más peso variables asociadas a la dinámica de la participación de las familias en el sistema escolar: asistencia, compromiso, motivación (Lee, 2009; Mier \& Rabell, 2002; Pineau \& Levy-Leboyer, 1980; Ready, 2010; Wager et al., 2010), en cómo los profesores aprovechan las potencialidades de los niños con sus diferentes capacidades (Condron, 2007; Froyum, 2010; Grødem, 2009), así como las posibilidades de continuidad de los estudios o capacitaciones (Boliver, 2011; Kohn, 2011). Inclusive, es posible observar cómo la trayectoria se diferencia según la capacidad del sistema parental de tranferir recursos para los proyectos de los jóvenes. En los sectores desfavorecidos, los hijos deben transferir recursos hacia la familia de origen manteniéndose así la desigualdad pese al aumento de capacitaciones y recursos (Albertini \& Radl, 2012; Chou, 2008; Powell \& Steelman, 1995; Spilerman \& Wolff, 2012; Stark \& Junsen, 2002).
En cierta forma, este recorrido acerca de la desigualdad y la infancia revela el fenómeno económico tras la reproducción de la sociedad y sus diferenciaciones sociales; sin embargo, es aún oscuro respecto a los fenómenos subjetivos involucrados en el mismo proceso. Entre los pocos estudios que hay al respecto, algunos dejan de manifiesto la instalación temprana de ciertos principios de distribución, en los que se observa claramente una tendencia a la no rectificación de una situación de desigualdad, si esta se presenta como dada naturalmente (Barreiro, 2009; Del Río \& Strasser, 2007; Olson, Dweck, Spelke, \& Banaji, 2011).

Con esto como antecedente, la pregunta de esta investigación fue icuáles son los fenómenos que desde los más tempranos tiempos diferencian subjetivamente a las clases sociales?

Los procesos tempranos de subjetivación han sido abordados desde el principio por la psicologia moderna, muchas veces con finalidades higienistas o de control social y otras tantas con la pretensión de dilucidar a partir de dicho conocimiento las claves para comprender los complejos procesos que se manifiestan en el mundo adulto (Burman, 1994). Por ello, todo intento que busque introducirse en la interpretación de las producciones infantiles, no puede sino referirse a los más importantes pensadores de la psicología clinica: Piaget, Wallon, Rey, Freud y en particular a Mahler, Klein, Bick, Gutton, Winnicott, etc., en el psicoanálisis.

En este trabajo de investigación, se incorporó la discusion de los distintos autores mencionados respecto a los aspectos que diagnósticamente se consideran en la observación clínica del juego infantil simbólico, para ello se proponen cinco dimensiones diagnósticas como elementos centrales para análisis en la investigación social:

1. La relación con el objeto-juguete: indaga aspectos relacionados con la percepción, juicio y sentido de realidad y las posibilidades del sujeto de actuar como agente de transformación ante dicho reconocimiento.

2. La relación de pares y con la autoridad (en términos de la protección y la norma): permite observar los procesos de identificación y dife- 
renciación que se producen en los tempranos tiempos de separación, individuación y definición de yo/no yo.

3. La experiencia corporal: habla de la instalación de los bordes físicos de la separación y diferenciación entre el sujeto y los otros.

4. Las reglas: permiten identificar la relación del sujeto con las normas, su capacidad de respetar un marco normativo que deviene de otro, de ser sujeto de la acción normativa y de comprender el origen y proyección de la ley en escenarios y relaciones particulares.

5. Los contenidos temáticos: dan cuenta de la compleja relación de elementos provenientes del mundo externo e interno del sujeto, sintetizada en una trama de juego particular. Esta dimensión evalúa la complejidad del mundo interno y los recursos simbólicos del sujeto para adicionar y subvertir el devenir de las historias de las que hace mímesis.

Pare implementar este diseño, se observó el juego de niños entre tres y cinco años en contextos no experimentales de distintas clases sociales. Se distinguieron dos modalidades de juego (juego ampliado y juego restringido1), las cuales podrían estar relacionadas con el desarrollo de mecanismos simbólicos que favorecen o dificultan el razonamiento sobre la reproducción social del orden y su desigualdad.

\section{Método}

Este es un estudio comparativo entre clases sociales $^{2}$, pues uno de los supuestos fundamentales es que

1 Las he nominado así con la finalidad de convocar mediante estos nombres el trabajo de Basil Bernstein, el cual es esclarecedor en esta materia, aunque desde una perspectiva vinculada a la educación y a la sociolingüística.

2 Los estudios de las "clases" se distinguen por quienes las definen en términos graduales y quienes lo hacen en términos relacionales. La clase, desde una perspectiva gradual, se refiere exclusivamente a los distintos niveles de ingreso de la población, mientras que la clase, en términos relacionales (marxistas y weberianos), designa la naturaleza de las relaciones sociales que subyacen al resultado distributivo. Este estudio se ubica entre quienes consideran las clases desde un análisis relacional marxista. En esta propuesta, se distingue entre dos grandes clases sociales organi- la sociedad construye mecanismos de introducción temprana de la ideología, que son la base de los mecanismos legitimantes y favorecedores de la reproducción social. La instituciones más relevantes en la transmisión de representaciones y que a través de sus prácticas inciden en la conformación de la identidad en la infancia, son: la familia (Milanich, 2009) y la escuela -en este caso la educación preescolar- (Bernstein, 1975).

Por ello, en el estudio empírico realizado en Santiago de Chile durante los años 2009 y 2010, participaron 5 espacios preescolares públicos y privados, 7 educadores preescolares, 11 madres y 10 niños.

El diseño cualitativo sustentado en las tradiciones comprensivas-interpretativas, de modo específico en aquellas que se vinculan al enfoque etnográfico y al enfoque interaccionista simbólico.

Se optó por un muestreo intencional de casos, utilizando la modalidad de "intención basada en criterios teóricos".

\section{Participantes}

Las familias participantes del estudio deben ser seleccionadas considerando la discusión en torno a clases sociales y movilidad social (Wright, 1997, 2010). Según los estudios al respecto ${ }^{3}$, se selec-

\footnotetext{
zadas en función de la propiedad de los medios de producción, lo cual es una característica de la propuesta analítica de Marx, sin embargo, él también propone en su análisis de los acontecimientos una concepción de clase histórica-descriptiva que permite identificar una diversidad de grupos sociales. Para el estudio de la sociedad contemporánea, han surgido bastantes propuestas que hacen operativo, desde un punto de vista más descriptivo, el panorama de las clases (Crompton, 1997); yo tomaré para la organización de este trabajo la propuesta de Wright (1998).

3 Esta estratificación se ha estudiado en el país tomando en cuenta fundamentalmente tres estudios: 1) la Encuesta de Ocupación y Desocupación que la Universidad de Chile realiza desde fines de la década de 1950 en el Gran Santiago; 2) la Encuesta de Presupuestos Familiares que ejecuta el Instituto Nacional de Estadísticas (INE) cada diez años, también para el Gran Santiago; 3) la Encuesta de Caracterización Socioeconómica Nacional (Casen) que efectúa periódicamente el Ministerio de Planificación (MIDEPLAN) y que es para todo el país. En relación con esta información, los estudios han establecido diferentes segmentos (quintiles) según los cuales se divide la población y con ello se proyectan tanto las políticas públicas como las sociales. Ninguno de estos estudios es propiamente una encuesta de ingresos, pero en ellos se consulta por esta variable. Cada uno tiene ventajas y tiene defectos para realizar la proyección, sin embargo, por ahora
} 
TABLA 1

Criterios teóricos para la selección de familias

\begin{tabular}{|c|c|c|c|c|}
\hline & Familia Clase baja & $\begin{array}{l}\text { Familia Clase Media } \\
\text { Emergente }\end{array}$ & $\begin{array}{l}\text { Familia Clase media } \\
\text { Tradicional }\end{array}$ & Familia Clase Alta \\
\hline Por ingreso & $\begin{array}{l}\text { Familias } \\
\text { pertenecientes al } \\
\text { quintil I y II. Es } \\
\text { decir, con un ingreso } \\
\text { monetario inferior a } \\
330.373\end{array}$ & $\begin{array}{l}\text { Familias } \\
\text { pertenecientes al } \\
\text { quintil III y IV. Es } \\
\text { decir, con un ingreso } \\
\text { monetario inferior a } \\
2.057 .759 \text { y superior a } \\
\text { los } 330.373 \text {. }\end{array}$ & $\begin{array}{l}\text { Familias } \\
\text { pertenecientes al } \\
\text { quintil III y IV. Es } \\
\text { decir, con un ingreso } \\
\text { monetario inferior a } \\
2.057 .759 \text { y superior a } \\
\text { los } 330.373 \text {. }\end{array}$ & $\begin{array}{l}\text { Familias } \\
\text { pertenecientes al } \\
\text { quintil V. Es decir, con } \\
\text { un ingreso monetario } \\
\text { superior a 2.057.759 }\end{array}$ \\
\hline Por ocupación & $\begin{array}{l}\text { Supervisores no } \\
\text { calificados } \\
\text { Empleados u obreros } \\
\text { calificados } \\
\text { Obreros } \\
\text { semicalificados } \\
\text { Servicios no } \\
\text { calificados } \\
\text { Obreros no calificados }\end{array}$ & \multicolumn{2}{|c|}{$\begin{array}{l}\text { Profesionales } \\
\text { Servicios } \\
\text { Calificados } \\
\text { Obreros Calificados } \\
\text { Directivos semicalificados } \\
\text { Directivos no calificados } \\
\text { Supervisores } \\
\text { Supervisores expertos o calificados }\end{array}$} & $\begin{array}{l}\text { Directivos } \\
\text { Directivos expertos o } \\
\text { calificados }\end{array}$ \\
\hline Por movilidad social & $\begin{array}{l}\text { Con permanencia } \\
\text { de al menos dos } \\
\text { generaciones en la } \\
\text { misma posición social. }\end{array}$ & $\begin{array}{l}\text { Primera generación } \\
\text { en dicha posición } \\
\text { social. }\end{array}$ & $\begin{array}{l}\text { Con permanencia } \\
\text { de al menos dos } \\
\text { generaciones en la } \\
\text { misma posición social. }\end{array}$ & $\begin{array}{l}\text { Con permanencia } \\
\text { de al menos dos } \\
\text { generaciones en la } \\
\text { misma posición social. }\end{array}$ \\
\hline $\begin{array}{l}\text { Con niños con } \\
\text { las siguientes } \\
\text { características }\end{array}$ & \multicolumn{4}{|c|}{$\begin{array}{l}\text { Niños entre los } 3 \text { y } 5 \text { años de edad, con un desarrollo que no haya sido afectado por } \\
\text { enfermedades crónicas, retardo mental o severos trastornos en el lenguaje }\end{array}$} \\
\hline
\end{tabular}

Fuente: elaboración propia.

cionaron participantes de acuerdo a los quintiles de ingreso referenciados por MIDEPLAN, pero se adicionaron otros dos criterios: el relativo a la ocupación, por considerarse que la formación educacional es uno de los elementos reconocido por los autores como una manera de apropiación de capital (Tilly, 2000; Wright, 1997, 1998) y el relativo a la movilidad social, por considerarse que en lo que refiere a la transmisión intergeneracional de las representaciones hay una importante diferencia entre quienes obtienen ingresos de una posición social más alta en primera generación que quienes sostienen la misma posición de clase generación tras generación (Rosanvallon \& Fitoussi, 1997). El cuarto y último criterio refiere a las características de los niños pertenecientes a las familias que participaron en el estudio (Tabla 1).

es la principal fuente de la cual se obtienen y corrigen estos datos para ofrecer un panorama cierto al respecto.
Los establecimientos preescolares fueron definidos segun dos criterios: 1) criterio territorial, es decir, mediante los datos de la Encuesta de Ocupación de la Universidad de Chile se localizaron las comunas en las que se concentran de manera relativamente homogénea las ocupaciones, 2) dependencia administrativa: con financiamiento estatal o particular privado (Tabla 2)

Se buscó desarrollar una entrevista a un educador por jardín infantil observado. En el caso de la clase baja, se entrevistaron tres, pues dos educadores en el jardín de Renca tenían contacto directo con los niños observados.

\section{Número de participantes}

Los instrumentos se aplicaron en distintos segmentos social a un numero total de 10 niños, 11 madres, 5 establecimientos educacionales y 7 educadores. 
TABLA 2

\begin{tabular}{lcccc}
\hline & Clase social baja & $\begin{array}{c}\text { Clase social } \\
\text { Media emergente }\end{array}$ & $\begin{array}{c}\text { Clase social Media } \\
\text { tradicional }\end{array}$ & Clase Social Alta \\
\hline Dependencia & $\begin{array}{c}\text { Con financiamiento } \\
\text { público o sin fines de } \\
\text { lucro (fundaciones, } \\
\text { ONG, etc.) }\end{array}$ & Privado & Privado & Privado \\
\hline Comuna & $\begin{array}{c}\text { Renca } \\
\text { Lo Espejo }\end{array}$ & Providencia & La Reina & Vitacura \\
\hline
\end{tabular}

Fuente: elaboración propia.

\section{Instrumentos}

Entrevista a la madre. Guiada por pauta de entrevista semiestructurada, se buscó explorar las siguientes dimensiones: formación educacional de la entrevistada, percepciones y prácticas referidas a la infancia chilena, la sociedad, el juguete y las clases sociales.

Observación al niño. Se desarrolló durante un mes en su casa. Guiada por pauta de observación que indagaba por: los juegos, objetos presentes en la escena, objetos utilizados, conductas no lúdicas, relación con semejantes, con alguna figura de autoridad, con el observador.

En los establecimientos de educación infantil se aplicaron los siguientes instrumentos:

Entrevista a la educadora. Guiada por pauta de entrevista semiestructurada, se buscó explorar las siguientes dimensiones: formación académica, experiencias vitales relacionadas con la profesión, caracterización de la institución en que trabaja, percepciones y prácticas referidas a la infancia chilena, la sociedad, el juego, el juguete y las clases sociales.

Observación. Realizada durante dos meses, una vez por semana, al interior del aula y en el patio. Guiada por pauta de observación que indagaba por: espacio de juego, características del juego, objetos presentes en la escena, objetos utilizados, conductas no lúdicas y prácticas educativas, relación con semejantes, con alguna figura de autoridad, con el observador.

\section{Limites}

La limitación fundamental está asociada al número de participantes. Siendo un universo acotado, solo es posible formular algunas ideas directrices sobre el tema.

\section{Técnicas de análisis-síntesis}

El análisis se basó en lo establecido en la literatura y en el corpus construido a partir de la observación de juego. Los elementos distinguidos como relevantes fueron los siguientes:

1. Las sensaciones y experiencias corporales de los niños, es decir sus movimientos en la escena del juego y de relación con los juguetes.

2. Las características de la relación entre el niño y las figuras protectoras y las variaciones en intensidad, tono y contenido de esta relación en la escena del juego, junto a la función de los juguetes en la expresión de dichas variaciones.

3. Los significados que el niño le adjudica a las figuras protectoras y las formas en que se manifiestan explícita o implícitamente y si el juguete cumple o no una función en esta comunicación.

4. El desarrollo de capacidades y su relación con los elementos del entorno (personas y objetos).

5. La reacción del entorno ante las demandas del niño durante el juego.

6. Las características de la relación entre el niño y los pares (niños, hermanos, primos, etc.) y las variaciones en intensidad, tono y contenido de esta relación en la escena del juego, junto a la función de los juguetes en la expresión de dichas variaciones.

7. La procedencia de los significados atribuidos a los objetos. 


\section{TABLA 3}

\begin{tabular}{|c|c|}
\hline Experiencia corporal & $\begin{array}{l}\text { Juego de manifestación de destrezas físicas con objeto-juguete. } \\
\text { Juego de manifestación de destrezas físicas sin objeto-juguete. } \\
\text { Juego de movimiento con propósito de descarga energética con objeto-juguete. } \\
\text { Juego de movimiento con propósito de descarga energética sin objeto-juguete. } \\
\text { Juego de contacto con bordes físicos de la escena con objeto-juguete. } \\
\text { Juego de contacto con bordes físicos de la escena sin objeto-juguete } \\
\text { Juego de contacto con pares con objeto-juguete. } \\
\text { Juego de contacto con pares sin objeto-juguete. } \\
\text { Juego de contacto con figura adulta significativa con objeto-juguete. } \\
\text { Juego de contacto con figura adulta significativa sin objeto-juguete. } \\
\text { Juego de evasión de contacto físico con objeto-juguete. } \\
\text { Juego de evasión de contacto físico sin objeto-juguete. }\end{array}$ \\
\hline Relación con la autoridad & $\begin{array}{l}\text { Juego de mímesis de actividades adultas con objeto-juguete. } \\
\text { Juego de mímesis de actividades adultas sin objeto-juguete. } \\
\text { Juego de mímesis de actividades adultas normativas con objeto-juguete. } \\
\text { Juego de mímesis de actividades adultas normativas sin objeto-juguete. } \\
\text { Juego de desafío a la autoridad con objeto-juguete. } \\
\text { Juego de desafío a la autoridad sin objeto-juguete. } \\
\text { Juego de convocatoria a la autoridad con objeto-juguete. } \\
\text { Juego de convocatoria a la autoridad sin objeto-juguete. } \\
\text { Juego de investimiento de la autoridad en el niño con objeto-juguete. } \\
\text { Juego de investimiento de la autoridad en el niño sin objeto-juguete. }\end{array}$ \\
\hline Relación con la protección & $\begin{array}{l}\text { Juego de introyección de actitudes protectoras con objeto-juguete. } \\
\text { Juego de introyección de actitudes protectoras sin objeto-juguete. } \\
\text { Juego de convocatoria a la protección adulta con objeto-juguete. } \\
\text { Juego de convocatoria a la protección adulta sin objeto-juguete. } \\
\text { Juego de socialización de conductas protectoras con objeto-juguete. } \\
\text { Juego de socialización de conductas protectoras sin objeto-juguete. }\end{array}$ \\
\hline Relación de pares & $\begin{array}{l}\text { Juego de competencia con objeto-juguete. } \\
\text { Juego de competencia sin objeto-juguete. } \\
\text { Juego solitario con objeto-juguete. } \\
\text { Juego solitario sin objeto-juguete. } \\
\text { Juego aislado con objeto-juguete. } \\
\text { Juego aislado sin objeto-juguete. } \\
\text { Juego colaborativo con objeto-juguete. } \\
\text { Juego colaborativo sin objeto-juguete. } \\
\text { Juego colaborativo sin objeto-juguete con liderazgo marcado. } \\
\text { Juego de fantasía compartida con objeto-juguete. } \\
\text { Juego de fantasía compartida sin objeto-juguete. } \\
\text { Juego de rivalización con objeto-juguete. } \\
\text { Juego de rivalización sin objeto juguete. }\end{array}$ \\
\hline Objetos-juguete & $\begin{array}{l}\text { Juego con la función propuesta por el objeto. } \\
\text { Juego que subvierte la propuesta representacional del objeto. } \\
\text { Juego que consiste en adaptar el objeto para crear juego. } \\
\text { Juego que consiste en adaptar el juego a las características del objeto. }\end{array}$ \\
\hline Reglas & $\begin{array}{l}\text { Juego que subvierte la norma del juguete. } \\
\text { Juego que subvierte la norma de los padres. } \\
\text { Juego que subvierte la norma de la institución. } \\
\text { Juego que subvierte las reglas del juego. } \\
\text { Juego de reglas. }\end{array}$ \\
\hline
\end{tabular}

Fuente: elaboración propia.

De acuerdo con estos elementos, se establecieron inicialmente las siguientes categorías, no excluyentes entre sí, es decir, los juegos observados pueden ser tabulados conteniendo varios elementos de una o más de las categorías propuestas.
Una vez analizado el corpus de datos obtenido en la observación de juegos, tanto en el espacio de educación infantil como en la casa, se agregó un nuevo grupo de categorías que resaltaron por su contenido temático. Estas fueron: 


\section{TABLA 4}

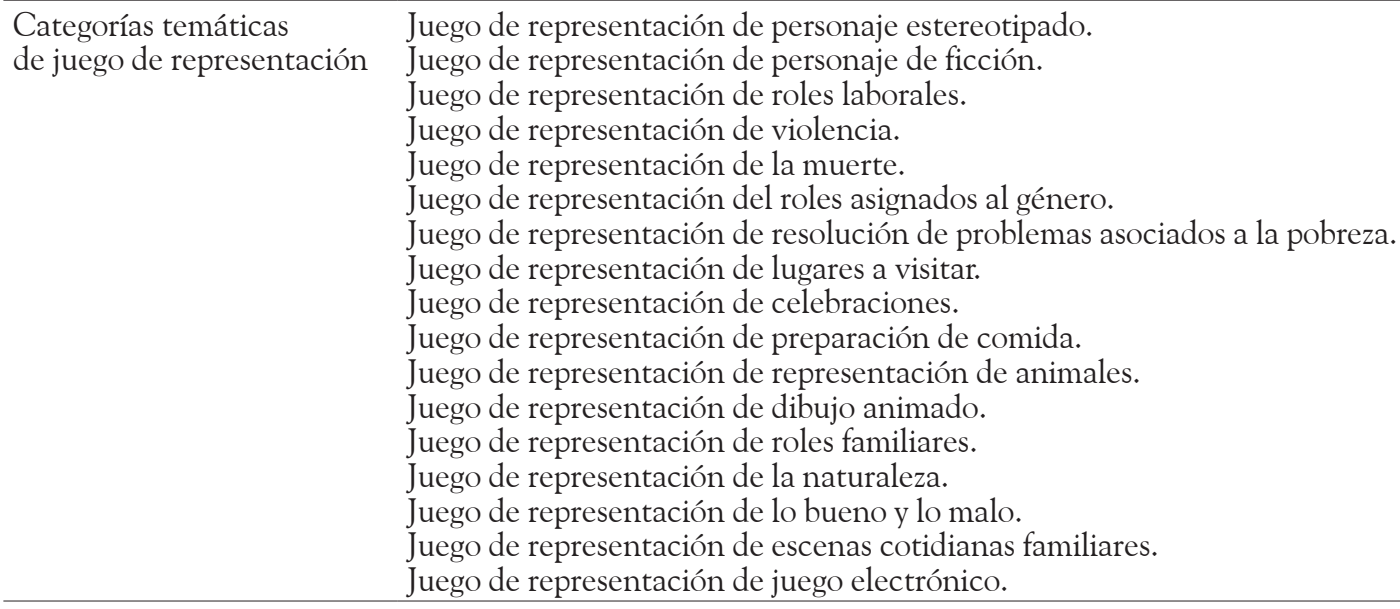

Fuente: elaboración propia.

Con estas categorías se procedió a analizar el conjunto de la información, segmentándolo por posición social y por localización en términos del marco institucional en el que se producen.

\section{Resultados}

\section{Modalidades de juego restringido y ampliado}

De acuerdo a los resultados obtenidos en la observación clínica de juego infantil fue posible distinguir fundamentalmente dos modalidades de juego que se diferencian radicalmente y que enuncian procesos de constitución subjetivos amparados en procesos socialización distintos.

Estas dos presentaciones de juego se distinguen de acuerdo a las dimensiones estudiadas y se presentan de manera diferenciada en los segmentos sociales altos y bajos de la sociedad. Refiriéndonos a la clasificación que Basil Bernstein plantea en relación con los códigos sociolingüísticos, nombraremos estas modalidades de juego como: juego ampliado y juego restringido ${ }^{4}$.

El juego ampliado se distingue por:

4 Claramente, hay diferencia entre hablar de códigos sociolingüísticos y hablar de la enunciación mediante juego. Sin embargo, en relación con la descripción de una determinada forma de percibir, otorgar sentido y actuar en la realidad, así como de vincularse con el contexto cultural, ambas manifestaciones simbólicas son bastante ricas y comparables en elementos.
1. En la relación con los objetos-juguetes, se manifiesta una plasticidad que implica la percepción ajustada a la realidad (reconoce la propuesta formal del objeto), pero puede asignarle diferentes sentidos, subordinando el objeto a la trama del juego que el niño quiere desarrollar.

2. En la relación con las instituciones, se manifiesta una sensibilidad en el juego que implica el reconocimiento de una diversidad de contextos, que convocan a su vez a una diversidad de comportamientos. La relación con las instituciones y con las personas no es rígida: la consideración de la demanda del otro, de la institución, así como de los semejantes introduce variables comportamentales en el juego. El niño tiene más de un patrón para relacionarse con los diferentes otros de la sociedad, se ubica rápidamente en torno a ello y despliega una conducta que considera apropiada.

3. La relación de pares puede fluir sobre distintas formas de agrupamiento, con liderazgos circunstanciales. Esto implica que la oportunidad de liderar un juego está presente para todos, no dependiendo de características exclusivamente de personalidad o de posesión de objetos.

4. Es un juego en el que confluyen distintas dimensiones de la experiencia corporal, donde la descarga de energía y emociones es un elemento vital, pero no el único. La experiencia corporal debe incluir el desarrollo de habilidades motrices de la zona de desarrollo próximo y el contacto 
afectivo con pares que permita la distinción sensible de los bordes corporales de cada uno.

5. La relación con la autoridad provee las referencias conductuales básicas para proteger y normar que luego son introyectadas y se transforman en insumos del investimiento de autoridad que recae sobre los niños en el contexto público y privado. Es decir, en esta modalidad de juego, los niños son capaces de convocar al otro en el lugar de protección, incorporan estas conductas entre sus referencias conductuales y luego son capaces de socializarlas para el cuidado de sí mismos y de los otros, desplazándose de su lugar de objeto de cuidados al de sujeto de cuidados.

6. Las temáticas dominantes de los juegos son heterogéneas y, si bien incorporan insumos para la fantasía proveniente de los medios de comunicación de masas, ingresan dentro de un conjunto más amplio de preocupaciones en los que es posible divisar cuestiones relacionadas con la identidad, la diferenciación y la problematización de los imperativos morales de la cultura.

7. Se acude a la forma de mímesis de conductas adultas, sin embargo, añade elementos originales a las escenas recreadas, incluyendo soluciones y objetos a la trama que provienen de un proceso de simbolización complejo y no de la mera repetición de conductas.

El juego restringido se distingue por:

1. En la relación con los objetos-juguetes se manifiesta una rigidez que implica la percepción ajustada a la realidad (reconoce la propuesta formal del objeto), pero no puede asignarle diferentes sentidos, subordinando el juego a la propuesta del objeto-juguete.

2. En la relación con las instituciones, no manifiesta una sensibilidad en el juego que implica el reconocimiento de una diversidad de contextos. La relación con las instituciones y con las personas es rígida: la consideración de la demanda del otro de la institución así como de los semejantes no introduce variables comportamentales en el juego. El niño tiene dificultades para relacionarse con los diferentes otros de la sociedad.
3. La relación de pares no puede fluir sobre distintas formas de agrupamiento, con liderazgos excesivamente rígidos, en los que la oportunidad de liderar un juego no está presente para todos, dependiendo esta de características exclusivamente de personalidad o de posesión de objetos.

4. En él no confluyen distintas dimensiones de la experiencia corporal. La descarga de energía y emociones es el único objetivo, o bien el contacto con pares es excesivamente violento. La presencia de juego cuya finalidad es constituir los bordes corporales a partir del contacto directo con los objetos del ambiente (paredes, piso, muebles) indica la dificultad de algunos niños de poder comprender dónde empieza y termina su cuerpo. Su juego se ubica en estadios del desarrollo mucho más temprano.

5. La relación con la autoridad se actualiza momento a momento, lo cual refleja la falta de introyección de aspectos normativos y protectores. La dependencia del adulto o de las figuras mayores presentes es singularmente alta, lo cual dificulta la posibilidad de crear juego, pues por momentos parece que el espacio transicional desaparece y la relación materno-filial vuelve a un punto de simbiosis correspondiente a las primeras etapas del desarrollo.

6. Las temáticas dominantes de los juegos son homogéneas e incorporan insumos para la fantasía, exclusivamente provenientes de los medios de comunicación de masas.

7. La recreación de escenas mediante el uso de mímesis no incorpora innovaciones que provengan de un mundo interno creativo y complejo. Se reduce a la encarnación de lo visto sin tramitación y por ello, sin cuestionamiento. Tiene más la forma de un adoctrinamiento que de una apropiación simbólica.

En este sentido, un modo predominante de juego ampliado se ubica en los sectores socioeconómicamente altos de la sociedad, mientras un modo predominante de juego restringido se ubica en los sectores vulnerables de la sociedad. Ello no quiere decir que no haya excepciones dentro de lo observado. Se trata más bien de construir un esquema 
conceptual que permita organizar la dinámica del juego dentro de sus posibilidades de favorecer procesos de constitución subjetiva en los que prevalezca la conciencia crítica de la posición del sujeto en un determinado contexto sociopolítico. Las pautas de crianza y de educación inciden en la forma en que estas coordenadas se ubican en los tiempos tempranos y se manifiestan en el juego infantil.

Se puede sintetizar ambas modalidades de juego en el siguiente cuadro (Tabla 5).

\section{Conclusiones}

La mayor parte de los elementos que se observan como distintivos en las modalidades de juego presentadas se puede identificar como relacionada con la constitución del espacio transicional conceptualizado por (Winnicott, 1970).

El juego ampliado, en términos winnicottianos, es la culminación del proceso de construcción del espacio transicional, el cual está en condiciones de permitir una superposición de dos zonas de juego, donde los niños pueden introducir su propio modo de jugar, aceptando o rechazando la introducción de ideas de los otros y del medio.

En el juego restringido, en cambio, se manifiestan conductas en las cuales se observa que algo del orden de la confianza en el ambiente no está suficientemente instalado, dificultando la expresión de lo mágico y lo omnipotente. Ello se manifiesta en la dificultad para jugar, ejercicio que supone en el imaginario del niño, la confianza en que la persona

\section{TABLA 5}

\begin{tabular}{|c|c|c|}
\hline Dimensión & Juego Ampliado & Juego Restringido \\
\hline Relación con el objeto & $\begin{array}{l}\text { 1.- Relación con el objeto de reconocimiento } \\
\text { de subversión y transformación en función } \\
\text { del juego. El juguete se adapta al juego. }\end{array}$ & $\begin{array}{l}\text { 1.- Relación con el objeto en la que } \\
\text { predomina la propuesta representacional del } \\
\text { objeto-juguete. El juego se adapta al juguete. }\end{array}$ \\
\hline $\begin{array}{l}\text { Experiencia } \\
\text { Espacial }\end{array}$ & $\begin{array}{l}\text { 2.- Es sensible al espacio en el que se } \\
\text { desarrolla. Cambia y se adapta a las } \\
\text { circunstancias. }\end{array}$ & $\begin{array}{l}\text { 2.- Es insensible al espacio en el que se } \\
\text { desarrolla. El niño juega a lo mismo en } \\
\text { cualquier escenario, no importando lo } \\
\text { adecuado de su conducta. }\end{array}$ \\
\hline Relación de pares & $\begin{array}{l}\text { 3.- Relación de pares con una dinámica } \\
\text { flexible y liderazgos contingentes. }\end{array}$ & $\begin{array}{l}\text { 3.- Relación de pares rígida, en la cual los } \\
\text { agrupamientos son fijos y estables. Los } \\
\text { liderazgos se definen de acuerdo a saber o } \\
\text { posesión. }\end{array}$ \\
\hline Experiencia corporal & $\begin{array}{l}\text { 4.- La experiencia corporal transita entre } \\
\text { la descarga de energía y sentimientos. La } \\
\text { diferenciación e identificación con los pares } \\
\text { se produce mediante el contacto afectivo y } \\
\text { cariñoso. }\end{array}$ & $\begin{array}{l}\text { 4.- La experiencia corporal se concentra en } \\
\text { la descarga de energía. La diferenciación } \\
\text { e identificación con los pares se produce } \\
\text { mediante un contacto violento y en } \\
\text { ocasiones se privilegia el contacto con los } \\
\text { bordes físicos (paredes, muebles, piso) al de } \\
\text { los pares. }\end{array}$ \\
\hline $\begin{array}{l}\text { Relación con la } \\
\text { autoridad Relación con } \\
\text { la protección }\end{array}$ & $\begin{array}{l}\text { 5. La relación con la autoridad evidencia } \\
\text { convocatoria, introyección y socialización de } \\
\text { las conductas protectoras y normativas. }\end{array}$ & $\begin{array}{l}\text { 5. La autoridad es permanentemente } \\
\text { convocada. Esto se expresa en un alto } \\
\text { nivel de dependencia de la figura materna, } \\
\text { inclusive en el borramiento del espacio } \\
\text { transicional y del juego. }\end{array}$ \\
\hline Dimensión creatividad & $\begin{array}{l}\text { 7. Las temáticas desarrolladas en los juegos } \\
\text { son heterogéneas y provienen de diversas } \\
\text { fuentes. }\end{array}$ & $\begin{array}{l}\text { 7. Las temáticas desarrolladas en los juegos } \\
\text { son homogéneas, provienen de la influencia } \\
\text { exacerbada de los medios de comunicación y } \\
\text { de la vida familiar cotidiana. }\end{array}$ \\
\hline $\begin{array}{l}\text { Dimensión mundo } \\
\text { interno }\end{array}$ & $\begin{array}{l}\text { 8. El contenido de los juegos incorpora forma } \\
\text { de mímesis, pero se añaden a las escenas } \\
\text { elementos originales que dan cuenta de } \\
\text { una elaboración y búsqueda de soluciones } \\
\text { amparadas en un proceso simbólico } \\
\text { complejo. }\end{array}$ & $\begin{array}{l}\text { 8. Las escenas que toman forma de } \\
\text { mímesis son reproducciones exactas de } \\
\text { las experiencias vividas y no incorporan } \\
\text { elementos originales que den cuenta de } \\
\text { un proceso de asociación y síntesis de un } \\
\text { simbolismo complejo. }\end{array}$ \\
\hline
\end{tabular}

Fuente: elaboración propia. 
amada está cerca y sigue estándolo en el recuerdo. Así, se instala una dificultad importante en el desarrollo que va desde los fenómenos transicionales al juego, de este al juego compartido, y de este a las experiencias culturales.

De acuerdo a los elementos teóricos planteados, es posible discriminar una cierta organización formal de las modalidades de juego que hemos descrito como ampliadas y restringidas. Ellas, siguiendo a Vygotsky (1995), hablan de una impregnación de los elementos socioculturales, de los cuales devendrá la posibilidad del niño de elaborar significados abstractos, separados de los objetos del mundo, que se concretiza en la formación de la imaginación.

En esta imaginación, que sería una función cognitiva superior para Vygotsky, es donde podemos ubicar también la capacidad reflexiva de los niños de reconocer su posición social en relación con otros y de subvertir los elementos contextuales con los que se encuentran identificados. Ello claramente estará relacionado con la posibilidad de poner en cuestión dicho lugar. Si la imaginación queda restringida a la pura apropiación de las reglas sociales, siendo estas además profundamente dependientes del mundo material y de la acción, difícilmente pueden incorporarse cuestionamientos que se orienten a la desnaturalización del orden social.

Así, el juego pierde su condición, descrita por Scheines (1998), de interrumpir el orden de la vida ordinaria, de destruirlo temporalmente, para fundar, en el vacío que queda en su lugar, el orden lúdico. Esta condición de interrupción, que G. Brougère (2005) explica como una relación con la realidad de segundo grado, es lo que permite que se produzcan experiencias de aprendizaje o elaboración consciente de conflictos. Esta condición de la actividad lúdica se produce tras la decisión performativa de participar en el juego, asumiendo que después de esta decisión se producirán muchas otras en las que se ampara el devenir del juego hacia caminos impredecibles. En el caso de los juegos restringidos, muchas veces algo queda suspendido en el carácter performativo de la decisión, es decir, el niño participa como si en el juego, que en resumen significa no jugándose nada, por ello las escenas observadas son fácilmente predecibles tanto en su desarrollo como en su fin.

En términos semánticos, también es posible leer esta diferencia social, tal como Gilles Brougère (2002) plantea, el juego se inscribe en una cultura que antecede su aparición como fenómeno de la infancia. Esta será co-construida en la interacción simbólica, entre el niño y las distintas influencias que se constituyen como bordes de la escena.

En este sentido, es esperable que en los segmentos sociales, donde tanto los adultos como los niños se encuentran privados de una heterogeneidad en cuanto expresiones culturales de toda clase, las experiencias de donde provienen las preguntas sobre lo que impacta, lo que angustia y lo que se desea repetir, se restringen a la recreación de la vida familiar, en su cotidianidad laboral (que está por demás expuesta a los niños de los sectores desfavorecidos), así como en sus vivencias de peligro y riesgo (violencia, peleas y amenazas) y la celebración de los cumpleaños (hito de festejo fundamental, por la capacidad que dicho evento tiene de poner al niño sobre-relieve para la mirada del otro).

Este, sin duda, es uno de los residuos más claramente visibles de la desigualdad, aunque no el más importante, pues la superación de esta diferencia encuentra solución rápidamente en la introducción de otras influencias, capaces de generar un impacto competitivo con las que dependen excesivamente del marco contextual.

Lo más significativo en este estudio está relacionado con la identificación de distintas estructuras formales para jugar, las cuales devienen de las prácticas parentales, inclusive más que de las educacionales.

Es decir, si bien es posible un análisis detallado de la modalidad de juego restringido y las razones que podrían estar implicadas en su instalación en los sectores más vulnerables de la sociedad, este no es el único elemento llamativo en este estudio. En él, es posible instalar algunas primeras hipótesis respecto a la forma en que se reproducen los comportamientos de clase que están relacionados con la reproducción y naturalización de un determinado hacer con el orden social. 
Las relaciones con pares, con el objeto-juguete, la diferenciación de espacios privados y públicos y las temáticas para ser elaboradas a través del comportamiento lúdico, pueden ser relacionadas con el lugar que cada niño va ocupar en el mediano plazo en la estratificación social. En cada una de estas posiciones, hay puntos ciegos que favorecen la instalación de mecanismos ideológicos, que a su vez pueden ser interpretados como mecanismos defensivos ante elementos de la realidad atentatorios contra los elementos más básicos en los que están fundamentadas las más primigenias sensaciones de seguridad.

\section{Referencias}

Albertini, M., \& Radl, J. (2012). Intergenerational transfers and social class: Inter-vivos transfers as means of status reproduction? Acta Sociologica, 55(2), 107123. http://dx.doi.org/10.1177/0001699311431596

Barreiro, A. V. (2009). La creencia en la justicia inmanente piagetiana: un momento en el proceso de apropiación de la creencia ideológica en un mundo justo. Psykhe, 18(1), 73-84.

Bernstein, B. (1975). Class, codes and control: Towards a theory of educational transmissions: Routledge \& Kegan Paul, Limited.

Boliver, V. (2011). Expansion, differentiation, and the persistence of social class inequalities in British higher education. Higher Education, 61(3), 229 242. http://dx.doi.org/10.1007/s10734-010-9374-y

Brougère, G. (2002). Jeu et loisir comme espaces d'apprentissages informels. Education et Sociétés, 10(2), 5-20. http://dx.doi.org/10.3917/es.010.0005

Brougère, G. (2005). Jouer/Apprendre. París: Economica.

Chou, K. -L. (2008). Parental repayment hypothesis in intergenerational financial transfers from adult children to elderly parents: Evidence from Hong Kong. Educational Gerontology, 34(9), 788-799. http://dx.doi.org/10.1080/03601270802095972

Condron, D. J. (2007). Stratification and educational sorting: Explaining ascriptive inequalities in early childhood reading group placement. Social Problems, 54(1), 139-160.

Del Río, M. F., \& Strasser, K. (2007). ¿Tienen los niños una teoría esencialista acerca de la pobreza? Psykhe, 16(2), 139-149.
Froyum, C. (2010). The reproduction of inequalities through emotional capital: The case of socializing low-income black girls. Qualitative Sociology, 33(1), 37-54. http://dx.doi.org/10.1007/s11133-009-9141-5

Grødem, A. S. (2009). The impact of poverty and immigrant background on children's school satisfaction: Evidence from Norway. International Journal of Social Welfare, 18(2), 193-201. http://dx.doi. org/10.1111/j.1468-2397.2008.00594.x

Janet, C. (2011). Inequality at birth: Some causes and consequences. The American Economic Review, 101(3), 1-22. doi: 10.1257/aer.101.3.1

Kohn, A. G. (2011). Oportunidades educativas y desigualdad: percepciones respecto a la incidencia de la educación en la estructura social. Espacio Abierto, 20(2), 239-266.

Lee, D. (2009). Three essays on the micro basis of socioeconomic inequality: The role of cognitive and noncognitive skills (Disertación doctoral, University of North Carolina, 2008). Dissertation Abstracts International Section A: Humanities and Social Sciences, 69(7-A), 9.

Magnuson, K. A., Meyers, M. K., Ruhm, C. J., \& Waldfogel, J. (2004). Inequality in preschool education and school readiness. American Educational Research Journal, 41(1), 115-157.

McLeod, J. D., Nonnemaker, J. M., \& Thiede Call, K. (2004). Income inequality, race, and child well-being: An aggregate analysis in the 50 United States. Journal of Health ES Social Behavior, 45(3), 249-264.

Mier, M. M., \& Rabell, C. (2002). Desigualdades en la escolaridad de los niños mexicanos. Revista Mexicana de Sociología, 64(3), 63-89.

Milanich, N. B. (2009). Children of fate: Childhood, class, and the state in Chile, 1850-1930. London: Duke University Press.

Niles, M. D., \& Peck, L. R. (2008). How poverty and segregation impact child development: Evidence from the Chicago Longitudinal Study. Journal of Poverty, 12(3), 306-332. http://dx.doi. org $/ 10.1080 / 10875540802198495$

Olson, K. R., Dweck, C. S., Spelke, E. S., \& Banaji, M. R. (2011). Children's responses to group-based inequalities: Perpetuation and rectification. Social Cognition, 29(3), 270-287. http://dx.doi.org/10.1521/ $\underline{\text { soco.2011.29.3.270 }}$ 
Özkan, Y. (2010). Interpersonal impact of the poverty on children. International Journal of Academic Research, 2(6), 172-179.

Pineau, C., \& Levy-Leboyer, C. (1980). Inégalité sociale et motivations scolaires. Enfance, 3, 135-156.

Powell, B., \& Steelman, L. C. (1995). Feeling the pinch: Child spacing and constraints on parental economic investments in children. Social Forces, 73(4), 1465-1486.

Ready, D. D. (2010). Socioeconomic disadvantage, school attendance, and early cognitive development: The differential effects of school exposure. Sociology of Education, 83(4), 271-286. http://dx.doi. org $/ 10.1177 / 0038040710383520$

Rosanvallon, P., \& Fitoussi, J. -P. (1997). La nueva era de las desigualdades.Buenos Aires: Ediciones Manantial.

Scheines, G. I. (1998). Juegos inocentes, juegos terribles. Buenos Aires: Eudeba.

Spilerman, S., \& Wolff, F. -C. (2012). Parental wealth and resource transfers: How they matter in France for home ownership and living standards. Social Science Research, 41(2), 207-223. http://dx.doi. org/10.1016/j.ssresearch.2011.08.002

Stark, O., \& Junsen, Z. (2002). Counter-compensatory inter-vivos transfers and parental altruism: Com- patibility or orthogonality? Journal of Economic Behavior E⿱ Organization, 47(1), 19-25.

Tilly, C. (2000). La desigualdad persistente. Buenos Aires: Ediciones Manantial.

Today, T. (2009). Poverty affects childhood. Therapy Today, 20(5), 5-5.

Vygotsky, L. (1995). Pensamiento y lenguaje. Barcelona: Paidós.

Wager, F., Hill, M., Bailey, N., Day, R., Hamilton, D., \& King, C. (2010). The impact of poverty on children and young people's use of services. Children $\mathcal{E}$ Society, 24(5), 400-412. http://dx.doi.org/10.1111/ j.1099-0860.2009.00236.x

Winnicott, D. W. (1970). Playing and reality. Harmondsworth, UK: Penguin.

Wright, E. O. (1997). Class counts: Comparative studies in class analysis. Cambridge: Cambridge University Press.

Wright, E. O. (1998). The debate on classes. London: Verso.

Wright, E. O. (2010). Preguntas a la desigualdad. Bogotá: Editorial Universidad del Rosario.

Yoshikawa, H., Aber, J. L., \& Beardslee, W. R. (2012). The effects of poverty on the mental, emotional, and behavioral health of children and youth. American Psychologist, 67(4), 272-284. http://dx.doi. org/10.1037/a0028015 